\title{
CORRIGENDUM
}

\section{Pharmacokinetics of 2,3,7,8-tetrachlorodibenzo-p-dioxin in Seveso adults and veterans of operation Ranch Hand}

\author{
JOEL E. MICHALEK, JAMES L. PIRKLE, LARRY L. NEEDHAM, DONALD G. PATTERSON Jr., \\ SAMUEL P. CAUDILL, RAM C. TRIPATHI AND PAOLO MOCARELLI
}

Journal of Exposure Analysis and Environmental Epidemiology (2002) 12, $44-53$ DOI: 10.1038/sj/jea/7500201

Since the publication of the above paper, the authors have identified an error in Table 3. A revised version of the table is given below.

Table 3. TCDD concentrations in the Seveso cohort.

\begin{tabular}{|c|c|c|c|c|}
\hline & \multicolumn{4}{|c|}{ TCDD concentration/years since exposure } \\
\hline & 0 & 0.27 & 3 & 16.35 \\
\hline \multicolumn{5}{|l|}{ (a) Males } \\
\hline & $(n=20)$ & $(n=11)$ & $(n=17)$ & $(n=24)$ \\
\hline Mean & 2833.3 & 2243.7 & 1311.4 & 195.4 \\
\hline Standard deviation & 2404.3 & 1574.7 & 1057.8 & 160.1 \\
\hline \multicolumn{5}{|l|}{ Percentiles } \\
\hline 0 & 77 & 358 & 96 & 30.7 \\
\hline 5 & 103.5 & 358 & 96 & 33.7 \\
\hline 50 & 2500 & 2010 & 1170 & 119.5 \\
\hline 95 & 7235 & 5910 & 3810 & 488 \\
\hline 100 & 9140 & 5910 & 3810 & 531 \\
\hline \multicolumn{5}{|l|}{ (b) Females } \\
\hline & $(n=21)$ & $(n=12)$ & $(n=15)$ & $(n=25)$ \\
\hline Mean & 2266.7 & 1622.2 & 1009.7 & 354.4 \\
\hline Standard deviation & 1909.0 & 1241.2 & 670.1 & 297.7 \\
\hline \multicolumn{5}{|l|}{ Percentiles } \\
\hline 0 & 103 & 375 & 97.2 & 35.4 \\
\hline 5 & 145 & 375 & 97.2 & 46.1 \\
\hline 50 & 1770 & 1515 & 933 & 271 \\
\hline 95 & 5730 & 4410 & 2400 & 871 \\
\hline 100 & 6320 & 4410 & 2400 & 878 \\
\hline \multicolumn{5}{|l|}{ (c) All } \\
\hline & $(n=41)$ & $(n=23)$ & $(n=32)$ & $(n=49)$ \\
\hline Mean & 2543.1 & 1919.4 & 1170 & 276.5 \\
\hline Standard deviation & 2156.4 & 1413.5 & 896.5 & 240.5 \\
\hline \multicolumn{5}{|l|}{ Percentiles } \\
\hline 0 & 77 & 358 & 96 & 30.7 \\
\hline 5 & 130 & 375 & 97.2 & 35.4 \\
\hline 50 & 1940 & 1720 & 1045 & 218 \\
\hline 95 & 5730 & 4410 & 3130 & 833 \\
\hline 100 & 9140 & 5910 & 3810 & 878 \\
\hline
\end{tabular}

